\title{
Association Between Basal Septal Hypertrophy And Left Ventricular Geometry In A Community Population
}

\section{Lan Gao}

Peking University First Hospital

Wei Ma ( $\square$ kmmawei@sina.com )

Peking University First Hospital

\section{Min Li}

Peking University First Hospital

\section{Ying Yang}

Peking University First Hospital

\section{Litong Qi}

Peking University First Hospital

\section{Baowei Zhang}

Peking University First Hospital

\section{Chonghui Wang}

Peking University First Hospital

\section{Yan Zhang}

Peking University First Hospital

\section{Yong Huo}

Peking University First Hospital

\section{Research Article}

Keywords: basal septal hypertrophy, left ventricular geometry, echocardiography

Posted Date: January 20th, 2022

DOI: https://doi.org/10.21203/rs.3.rs-1230429/v1

License: (c) (i) This work is licensed under a Creative Commons Attribution 4.0 International License. Read Full License 


\section{Abstract}

Background: In this study, we examined the relationship between basal septal hypertrophy (BSH) and left ventricular (LV) geometry in a Beijing community population.

Methods: The clinical and echocardiographic data of 1,032 participants from a community in Beijing were analyzed. BSH was defined as a basal interventricular septal thickness of $\geq 14 \mathrm{~mm}$ and a basal septal thickness/mid-septal thickness of $\geq 1.3 \mathrm{~mm}$. Based on their echocardiographic characteristics, patients were described as having a normal geometry, concentric remodeling, concentric hypertrophy, or eccentric hypertrophy. Multivariate logistic regression was used to analyze the relationship between $\mathrm{BSH}$, LV mass index (LVMI), and relative wall thickness (RWT).

Results: The prevalence of BSH was 7.4\%. Basal interventricular septal thickness, LV posterior wall thickness, and RWT were greater, while LVMI and LV end-diastolic dimension were lower in the BSH group than in the non-BSH group $(p<0.05)$. The BSH group accounted for the highest proportion of patients with concentric remodeling. A multivariate regression analysis showed that BSH independently correlated with RWT (odds ratio $3.874,95 \%$ confidence interval 1.983-7.571, $\mathrm{p}<0.05$ ), but not with LVMI (odds ratio $0.137,95 \%$ confidence interval 0.018 $1.017, p=0.052)$. There was no interaction between BSH and each covariate, including age, body mass index, sex, diabetes mellitus, coronary heart disease, stroke, and smoking.

Conclusions: In this study, BSH independently correlated with RWT. There was no significant difference in BSH or RWT between the BSH group and the non-BSH group.

\section{Background}

The prevalence of basal septal hypertrophy (BSH) is approximately $10 \%$ in the general population [1]. However, the prevalence of $\mathrm{BSH}$ varies by definition, age group, and comorbidities. For example, $\mathrm{BSH}$ is commonly observed in older individuals. A previous study reported that BSH rising to $18 \%$ in older individuals, while the prevalence of BSH in hypertensive cohorts is approximately $20 \%$ [2].

BSH is not independently associated with an adverse cardiovascular prognosis [2]. However, some studies have demonstrated that BSH is an early manifestation of hypertension. Therefore, self-measured blood pressure and ambulatory blood pressure monitoring should be performed in all patients to improve the detection of hypertension [3]. Significant BSH is associated with left ventricular (LV) outflow tract obstruction and heart failure with preserved ejection fraction [1, 4], as well as with impaired LV diastolic function [2]. Moreover, LV geometry is closely associated with cardiovascular disease, especially in patients with hypertension [5]. However, few studies have evaluated the relationship between BSH and LV geometry. Therefore, we aimed to explore the relationship between $\mathrm{BSH}$ and LV geometry in a Beijing community population.

\section{Methods}

\subsection{Population}

All residents who lived in the Shi Jing Shan District of Beijing and who were aged $>40$ years were invited to participate. The investigation methods have been published previously [6]. Of 5,593 subjects, 1,069 volunteered to participate. The investigation started in 2004 and ended in 2005. Participants who underwent echocardiography 
were recruited, while participants who had regional wall movement abnormalities, moderate or severe aortic valve stenosis, rheumatic heart disease, or congenital heart disease were excluded. Finally, 1,032 participants were included. The study was approved by the institutional review board of Peking University First Hospital, and informed consent was obtained from all participants.

\subsection{Definition of cardiovascular risk factors and disease}

The methods used to measure height, weight, blood pressure, heart rate, fasting blood glucose, oral glucose tolerance, and blood lipid concentrations have been described previously [7]. Current smokers and participants with a history of smoking were defined as smokers. Hypertension was defined as a systolic blood pressure of $\geq 140$ $\mathrm{mmHg}$ and/or a diastolic blood pressure of $\geq 90 \mathrm{mmHg}$ or a history or usage of antihypertensive drugs. Diabetes mellitus was diagnosed according to each participant's history. Participants with a fasting blood glucose concentration of $\geq 7.0 \mathrm{mmol} / \mathrm{L}$ and a 2-hour glucose concentration of $\geq 11.1 \mathrm{mmol} / \mathrm{L}$ were also defined as having diabetes mellitus. Stroke, including intracerebral hemorrhage, cerebral infarction, and transient ischemic attack, was defined by the patient's history. A history of myocardial infarction, percutaneous coronary intervention, and coronary artery bypass grafting were all included in coronary heart disease (CHD).

\subsection{Echocardiography}

Echocardiography was performed using a 3-MHz transducer and an ultrasound system (Vivid-7; General Electric). According to previously published guidelines [8], standard images were collected and stored. One experienced clinician who was blinded to the clinical picture of the participants measured the echocardiography parameters at the central laboratory of Peking University First Hospital.

Basal interventricular septal (IVS) thickness, middle IVS thickness, and LV posterior wall thickness (PWT) were measured at end-diastole. LV end-diastolic dimension (LVEDD) and LV end-systolic dimension were also measured. LV mass (LVM) was calculated as follows: $L V M=0.8 \times 1.04 \times\left([P W T d+S W T d+L V I D d]^{3}-[L V I D d]^{3}\right)+0.6 \mathrm{~g}$, where PWTd and SWTd are the posterior and septal wall thicknesses at end-diastole, respectively, and LVIDd is the LV dimension at end-diastole. LVM index (LVMI) was then calculated, as previously described [8].

Relative wall thickness (RWT) was calculated using the following formula: $(2 \times$ LV PWT) $\div$ LVEDD. An LVMI of $\leq 115 \mathrm{~g} / \mathrm{m}^{2}$ and an RWT of $\leq 0.42$ were defined as a normal geometry, an LVMI of $\leq 115 \mathrm{~g} / \mathrm{m}^{2}$ and an RWT of $>0.42$ were defined as concentric remodeling, an LVMI of $>115 \mathrm{~g} / \mathrm{m}^{2}$ and an RWT of $>0.42$ were defined as concentric hypertrophy, and an LVMI of $>115 \mathrm{~g} / \mathrm{m}^{2}$ and an RWT of $\leq 0.42$ were defined as eccentric hypertrophy [8].

BSH was defined when all three of the following criteria were fulfilled: (1) a basal IVS thickness of $\geq 14 \mathrm{~mm}$; (2) a basal septal thickness/mid-septal thickness of $\geq 1.3 \mathrm{~mm}$; and (3) no wall motion abnormalities or scarring in the middle septum that could result in isolated septal thickening.

\subsection{Statistical analysis}

Measurement data are presented as mean \pm standard deviation, while count data are presented as percentages. Continuous data were compared between the two groups using the Student's t-test. Categorical data were compared between the two groups using the chi-square test. Multivariate logistic regression was used to analyze the relationship between $\mathrm{BSH}, \mathrm{LVMI}$, and RWT, adjusting for age, sex, body mass index (BMI), hypertension, diabetes mellitus, systolic blood pressure, diastolic blood pressure, and heart rate. Subgroup analyses and interaction tests were used to examine the relationship between BSH and RWT according to age ( $<60$ years and 
$\geq 60$ years), sex (male and female), BMI ( $<24 \mathrm{~kg} / \mathrm{m}^{2}$ and $\geq 24 \mathrm{~kg} / \mathrm{m}^{2}$ ), diabetes mellitus (yes or no), CHD (yes or no), stroke (yes or no), and smoking status (yes or no). A two-sided p value of $<0.05$ was considered statistically significant for all tests. All analyses were performed using SPSS, version 13.0 for Windows (IBM Corp., Armonk, NY, USA).

\section{Results}

The general characteristics of the participants are shown in Table 1. The mean age of the participants was $64.02 \pm$ 9.00 years, and $51.8 \%$ of the participants were male. The prevalence of $\mathrm{BSH}$ was $7.4 \%$. Participants in the BSH group were older and had a higher fasting blood glucose concentration. The prevalence of diabetes mellitus was also higher in the BSH group than in the non-BSH group $(p<0.05)$.

Table 1

General characteristics of participants.

\begin{tabular}{|c|c|c|c|c|c|}
\hline & $\begin{array}{l}\text { Number } \\
\text { (\%) }\end{array}$ & Age(years) & $\begin{array}{l}\text { Sex(male, } n \text {, } \\
\%)\end{array}$ & $\begin{array}{l}\text { BMI } \\
\left(\mathrm{kg} / \mathrm{m}^{2}\right)\end{array}$ & $\begin{array}{l}\text { SBP } \\
(\mathrm{mmHg})\end{array}$ \\
\hline Total & 1032 & $64.02 \pm 9.00$ & $535(51.8)$ & $25.87 \pm 3.39$ & $134.02 \pm 18.03$ \\
\hline BSH & $76(7.4)$ & $66.18 \pm 8.36^{a}$ & $43(56.6)$ & $26.39 \pm 3.42$ & $137.23 \pm 19.29$ \\
\hline \multirow{2}{*}{$\begin{array}{l}\text { Non- } \\
\text { BSH }\end{array}$} & $956(92.6)$ & $63.85 \pm 9.03$ & 492(51.5) & $25.83 \pm 3.39$ & $133.76 \pm 17.91$ \\
\hline & $\begin{array}{l}\text { DBP } \\
(\mathrm{mmHg})\end{array}$ & $\mathrm{TC}(\mathrm{mmol} / \mathrm{l})$ & $\mathrm{TG}(\mathrm{mmol} / \mathrm{L})$ & $\begin{array}{l}\text { Fast } \\
\text { Glucose }(\mathrm{mmol} / \mathrm{L})\end{array}$ & $\begin{array}{l}\text { OGTT } 2 \mathrm{~h} \\
\text { Glucose(mmol/L) }\end{array}$ \\
\hline Total & $80.08 \pm 10.93$ & $5.29 \pm 1.03$ & $2.32 \pm 1.95$ & $6.37 \pm 2.25$ & $8.06 \pm 3.52$ \\
\hline BSH & $80.70 \pm 10.24$ & $5.17 \pm 0.84$ & $2.11 \pm 1.34$ & $7.17 \pm 3.03^{a}$ & $8.00 \pm 3.37$ \\
\hline \multirow{2}{*}{$\begin{array}{l}\text { Non- } \\
\text { BSH }\end{array}$} & $80.03 \pm 10.99$ & $5.30 \pm 1.05$ & $2.33 \pm 1.99$ & $6.31 \pm 2.13$ & $8.95 \pm 5.23$ \\
\hline & $\begin{array}{l}\text { Hypertension } \\
(\%)\end{array}$ & $\begin{array}{l}\text { Stroke } \\
(\%)\end{array}$ & $\begin{array}{l}\text { CHD } \\
(\%)\end{array}$ & $\begin{array}{l}\text { Diabetes } \\
(\%)\end{array}$ & $\begin{array}{l}\text { Smoking } \\
\text { (\%) }\end{array}$ \\
\hline Total & $824(79.8)$ & $169(16.4)$ & $130(12.6)$ & $305(29.6)$ & $366(35.5)$ \\
\hline BSH & $64(84.2)$ & 13(17.1) & $8(10.5)$ & $35(46.1)^{a}$ & $30(39.5)$ \\
\hline $\begin{array}{l}\text { Non- } \\
\text { BSH }\end{array}$ & $760(79.5)$ & 156(16.3) & $122(12.8)$ & $270(28.2)$ & $464(48.5)$ \\
\hline \multicolumn{6}{|c|}{$\begin{array}{l}\text { BSH, basal septal hypertrophy; BMI, body mass index; SBP, systolic blood pressure; DBP, diastolic blood } \\
\text { pressure; TC, total cholesterol; TG, triglyceride; OGTT, oral glucose tolerance test; CHD, coronary heart disease }\end{array}$} \\
\hline
\end{tabular}

The echocardiographic parameters of the participants are shown in Table 2. Compared with levels in the non-BSH group, basal IVS thickness, LV PWT, and RWT were greater, while LVMI and LVEDD were lower in the BSH group ( $p<$ 0.05). The BSH group accounted for the highest proportion of participants with concentric remodeling, with 
approximately $84.2 \%$ of participants being from the BSH group and $48.7 \%$ of participants being from the non-BSH group $(p<0.05)$.

Table 2

Echocardiographic parameters of participants.

\begin{tabular}{|c|c|c|c|c|c|c|}
\hline & IVSB(mm) & IVSM(mm) & LVPW(mm) & LVMI $\left(\mathrm{g} / \mathrm{m}^{2}\right)$ & LVEDD(mm) & LVEF(\%) \\
\hline Total & $10.05 \pm 2.23$ & $9.44 \pm 1.33$ & $9.22 \pm 0.73$ & $77.65 \pm 17.42$ & $43.14 \pm 5.21$ & $68.84 \pm 8.65$ \\
\hline $\mathrm{BSH}$ & $15.03 \pm 1.34^{a}$ & $9.65 \pm 0.81$ & $9.51 \pm 0.73^{a}$ & $68.99 \pm 13.35^{a}$ & $39.43 \pm 5.26^{a}$ & $68.76 \pm 8.49$ \\
\hline \multirow[t]{2}{*}{ Non-BSH } & $9.66 \pm 1.47$ & $9.42 \pm 1.36$ & $9.20 \pm 0.72$ & $78.34 \pm 17.52$ & $43.43 \pm 5.09$ & $68.84 \pm 8.67$ \\
\hline & $\mathrm{LAD}(\mathrm{mm})$ & RWT & $N G(n, \%)$ & $\mathrm{CR}(\mathrm{n}, \%)$ & $\mathrm{CH}(\mathrm{n}, \%)$ & $\mathrm{EH}(\mathrm{n}, \%)$ \\
\hline Total & $35.00 \pm 12.65$ & $0.43 \pm 0.06$ & $428(41.5)$ & $530(51.4)$ & $26(2.5)$ & $48(4.7)$ \\
\hline $\mathrm{BSH}$ & $39.20 \pm 4.43$ & $0.49 \pm 0.08^{a}$ & $11(14.5)^{\mathrm{a}}$ & $64(84.2)$ & $0(0)$ & $1(1.3)$ \\
\hline Non-BSH & $34.70 \pm 4.13$ & $0.43 \pm 0.06$ & $417(43.6)$ & $466(48.7)$ & $26(2.7)$ & $47(4.9)$ \\
\hline \multicolumn{7}{|c|}{$\begin{array}{l}\text { BSH, basal septal hypertrophy; IVSB, basal interventricular septum; IVSM, middle interventricular septum; LVPW, } \\
\text { left ventricular posterior wall thickness; LVMI, left ventricular mass index; LVEDD, left ventricular end-diastolic } \\
\text { dimension; LVEF, left ventricular ejection fraction; LAD, left atrial diameter; RWT, relative wall thickness; NG, } \\
\text { normal geometry; CR, concentric remodeling; CH, concentric hypertrophy; EH, eccentric hypertrophy. }\end{array}$} \\
\hline
\end{tabular}

A multivariate regression analysis showed that BSH independently correlated with RWT (odds ratio [OR] 3.874, 95\% confidence interval [CI] 1.983-7.571, p < 0.05), but not with LVMI (OR 0.137, 95\% Cl 0.018-1.017, p = 0.052) after adjusting for age, sex, BMI, hypertension, diabetes mellitus, systolic blood pressure, diastolic blood pressure, and heart rate. LVMI was also adjusted when studying the relationship between BSH and RWT. Detailed information is presented in Table 3. The results of the subgroup analysis showed that there were no interactions between BSH and any covariates, including age, BMI, sex, diabetes mellitus, CHD, stroke, and smoking. The details are shown in Figure 1. 
Table 3

Multivariate logistic regression analysis of $\mathrm{BSH}, \mathrm{LVMI}$, and RWT

\begin{tabular}{|c|c|c|c|c|c|c|c|c|}
\hline & \multicolumn{4}{|l|}{ LVMI } & \multicolumn{4}{|l|}{ RWT } \\
\hline & Crude OR(95\%Cl) & $P$ & $\begin{array}{l}\text { Adjusted } \\
\text { OR(95\%Cl })^{a}\end{array}$ & $P$ & $\begin{array}{l}\text { Crude } \\
\text { OR(95\%Cl) }\end{array}$ & $P$ & $\begin{array}{l}\text { Adjusted } \\
\text { OR( }(95 \% \mathrm{Cl})^{\mathrm{b}}\end{array}$ & $\mathrm{P}$ \\
\hline \multirow[t]{2}{*}{$\mathrm{BSH}$} & \multirow[t]{2}{*}{$1.161(0.022 .1 .177)$} & \multirow[t]{2}{*}{0.072} & 0.137 & \multirow[t]{2}{*}{0.052} & \multirow{2}{*}{$\begin{array}{l}5.030(2.680, \\
9.439)\end{array}$} & \multirow[t]{2}{*}{0.00} & 3.874 & \multirow[t]{2}{*}{0.00} \\
\hline & & & $\begin{array}{l}(0.018, \\
1.017)\end{array}$ & & & & $\begin{array}{l}\text { (1.983, } \\
7.571)\end{array}$ & \\
\hline
\end{tabular}

${ }^{a}$ Adjusted for age, sex, body mass index, hypertension, diabetes mellitus, systolic blood pressure, diastolic blood pressure, and heart rate.

${ }^{\mathrm{b}}$ Adjusted for age, sex, body mass index, hypertension, diabetes mellitus, systolic blood pressure, diastolic blood pressure, heart rate, and LVMI.

BSH, basal septal hypertrophy; LVMI, left ventricular mass index; RWT, relative wall thickness

\section{Discussion}

BSH detected by routine echocardiography is prominent in older individuals. In our study, the majority of participants with concentric remodeling were from the BSH group. BSH was independently correlated with RWT, but not with LVMI. The subgroup analysis showed no interaction between BSH and age, sex, BMI, diabetes mellitus, CHD, stroke, or smoking status.

The mechanism leading to BSH may be associated with Laplace's law. The larger the vessel radius, the larger the wall tension required to withstand pressure. The basal IVS has the largest radius in the human heart; thus, it would be expected to experience the greatest inward wall stress. The basal septum is the last part of the ventricle to be electrically activated, and contractions from other myocardial segments further increase wall stress in the basal septum. Moreover, the load created by pressure from the right ventricle exerts additional stress on the septum [1]. Longitudinal shortening is more pronounced in the basal IVS than at other sites in patients with hypertension [9].

Previous studies have indicated that the basal IVS is locally thickened in many patients with essential hypertension, which is recognized early in these subjects [10]. This localized thickening decreases in response to antihypertensive treatment [11]. Thus, BSH might be valuable to detect masked hypertension in the general population [3]. Central blood pressure correlates with basal SWT, but not with mid-SWT [12]. Thus, stricter blood pressure management and hypertension screening should be conducted in patients with BSH. A previously published cohort study showed that hypertensive subjects with BSH were older than non-BSH subjects and had a higher BMI and systolic blood pressure [13]. In our study, age and systolic blood pressure tended to be higher in patients with $\mathrm{BSH}$ than in those without, although the difference was not significant. There was no difference in the prevalence of hypertension between the BSH group and the non-BSH group, which may be because the overall population was older and the prevalence of hypertension was higher in our study.

A previous cohort study showed that hypertensive patients with BSH had a higher LV ejection fraction and lower LV end-diastolic and end-systolic volumes, while no significant differences were observed in left atrial size between the two groups [13]. In the present study, we found that LVEDD was lower in patients with BSH, while left atrial diameter was not significantly different. Unlike previous studies, the LVMI in the BSH group was lower than in the 
non-BSH group in our study, which may be related to the baseline level of the included populations. Early clinical studies suggested that BSH is related to LV diastolic function [2]. Another study showed that BSH is less likely to cause increased LV stiffness without LV hypertrophy [14], but that it can affect LV diastolic function during stress [15]. BSH is related to cardiac function in patients with hypertension with well-controlled blood pressure. Basal and mid-posterior wall systolic deformation, LV diastolic function, and left atrial function are decreased in these patients [13]. Thus, such patients are prone to heart failure with preserved ejection fraction.

$\mathrm{BSH}$ is also common in patients with severe aortic stenosis. This result suggests that an increase in afterload can cause BSH in patients with aortic stenosis, which is similar to patients with hypertension. A retrospective study using comprehensive echocardiography analyzed patients undergoing surgical aortic valve replacement (SAVR) or transcatheter aortic valve replacement (TAVR). The study showed that SAVR improved BSH, while TAVR did not. One of the mechanisms of BSH might be augmented compression of the basal IVS by a longitudinally elongated ascending aorta. SAVR involves incision and suture of the anterior wall of the ascending aorta; thus, the elongated aorta is shortened during SAVR. Aortic wall shortening may exert a superiorly directed force on the basal IVS, causing the myocardium to be less compressed and therefore less thick, improving basal IVS contraction. The effects of SAVR on BSH may not be expected from TAVR, given that TAVR does not involve wall shortening [16]. Patients with BSH are more likely to undergo postdilation during the TAVR procedure, and BSH is not associated with conduction outcomes after TAVR in patients without preexisting pacemakers [17]. This may contribute to LV outflow tract obstruction [18]. Thus, when TAVR is performed in patients with severe aortic stenosis, attention should be given to $\mathrm{BSH}$ to reduce the chance of missing a diagnosis of LV outflow tract.

In the present study, concentric remodeling was the most frequent LV geometry in patients with BSH. A retrospective analysis of a large clinical population $(n=35,602)$ showed an abnormal LV geometry in $46 \%$ of patients, with concentric remodeling present in $35 \%$ of patients $(n=12,362)$ and LV hypertrophy present in $11 \%$ of patients $(n=3,958)$ [5]. Patients with hypertension had race-related differences in LV geometry and RWT. A descriptive study previously reported that Africans exhibited a greater IVS thickness and RWT than Caucasians [19]. A study with a mean follow-up period of 2.5 years assessed the effect of potential changes in cardiac structure and found that 1,610 patients (45\%) demonstrated no change in LV geometry and maintained a pattern of concentric remodeling, 439 patients (12\%) progressed to LV hypertrophy, and 1,567 patients (43\%) converted to a normal LV geometry. There was a strong relationship between an abnormal LV geometry and all-cause mortality. Patients with concentric remodeling and LV hypertrophy (eccentric and concentric LV hypertrophy) exhibited considerably higher mortality than patients with a normal LV geometry $(8.7 \%, 8.4 \%$, and $10.4 \%$ vs. $4.4 \%$, respectively, $p<0.0001$ ) [5]. An American population-based case-control study showed that concentric remodeling is associated with stroke risk [20]. A prospective study showed that all-cause mortality was significantly more likely in patients with concentric remodeling (hazard ratio 1.417, 95\% Cl 1.045-1.920) [21]. Therefore, follow-up and risk factor control of patients with concentric remodeling should be strengthened to reduce the occurrence of cardiovascular events, but we did not pay sufficient attention to LV geometry.

In this study, BSH independently correlated with RWT. A previous study showed that an increase in RWT is a strong independent predictor of mortality [5]. RWT significantly increases stroke risk, but no interactions have been detected between RWT and LVM [20]. A prospective study showed that RWT is an independent predictor of allcause and cardiovascular mortality in patients who experience ischemic stroke, whereas the association between LVMI and all-cause death is not significant [21]. In the present study, hypertensive patients with BSH demonstrated a greater RWT and accounted for the highest proportion of patients with concentric remodeling. Therefore, if LV geometry is routinely measured in clinical practice, cardiovascular risk in patients with BSH may be increased. 
Our study has several limitations that should be noted. First, because of the cross-sectional study design, a causal relationship between BSH and LV geometry could not be determined. Prospective studies examining whether BSH is predictive of LV geometry and cardiovascular disease are required. Second, the majority of patients were aged $>40$ years; thus, our findings may not reflect the characteristics of $\mathrm{BSH}$ in a younger population.

\section{Conclusions}

Our study found that patients with BSH accounted for the highest proportion of patients with concentric remodeling. Furthermore, BSH independently correlated with RWT. There was no significant difference in BSH or RWT between the different subgroups. Patients who demonstrate BSH on echocardiography should undergo stricter cardiovascular risk factor control, especially for hypertension.

\section{Abbreviations}

BSH: basal septal hypertrophy

LVMI: left ventricular mass index

RWT: relative wall thickness

CHD: coronary heart disease

IVS: interventricular septum

RWT: relative wall thickness

\section{Declarations}

\section{Ethics approval and consent to participate}

The project has been approved by The Committees of Peking University First Hospital (Approval No: 2020Y328). Informed consent from participants was waived by the Peking University First Hospital Human Research Ethics Committee. All the study procedures were conducted following the Declaration of Helsinki.

\section{Consent for publication}

Not applicable.

\section{Availability of data and materials}

The datasets generated and analysed during the current study are not publicly available due data security issues, but are available from the corresponding author on reasonable request.

\section{Competing interests}

The authors declare that they have no conflicts of interest.

\section{Funding statement}


This study was financially supported by the National Key Research and Development Program of China (Project No. 2021YFC2501100, Subject No. 2021YFC2501106).

\section{Authors' contributions}

Manuscript drafting: Lan Gao \& Wei Ma; conception: Wei Ma \& Lan Gao; study design: Wei Ma \& Baowei Zhang; interpretation of data: Wei Ma, Min Li, \& Ying Yang; analysis: Wei Ma \& Min Li; data acquisition: Wei Ma, Chonghui Wang, Litong Qi, Lan Gao, \& Ying Yang; supervision: Wei Ma, Yan Zhang, \& Yong Huo.

The author(s) read and approved the final manuscript.

\section{Acknowledgments}

None.

\section{Author details}

1. Department of Cardiovascular Disease, Peking University First Hospital

2. Key Laboratory of Molecular Cardiovascular Sciences (Peking University), Ministry of Education

3. Echocardiography Core Lab, Institute of cardiovascular Disease at Peking University First Hospital

\section{References}

1. Kelshiker MA, Mayet J, Unsworth B, Okonko DO. Basal septal hypertrophy. Current cardiology reviews. 2013;9:325-30.

2. Diaz T, Pencina MJ, Benjamin EJ, Aragam J, Fuller DL, Pencina KM, et al. Prevalence, clinical correlates, and prognosis of discrete upper septal thickening on echocardiography: the Framingham Heart Study. Echocardiography (Mount Kisco, NY). 2009;26:247-53.

3. Gaudron PD, Liu D, Scholz F, Hu K, Florescu C, Herrmann S, et al. The septal bulge--an early echocardiographic sign in hypertensive heart disease. Journal of the American Society of Hypertension: JASH. 2016;10:70-80.

4. Pearson AC. The evolution of basal septal hypertrophy: From benign and age-related normal variant to potentially obstructive and symptomatic cardiomyopathy. Echocardiography (Mount Kisco, NY). 2017;34:1062-72.

5. Milani RV, Lavie CJ, Mehra MR, Ventura HO, Kurtz JD, Messerli FH. Left ventricular geometry and survival in patients with normal left ventricular ejection fraction. The American journal of cardiology. 2006;97:959-63.

6. Zhang L, Zuo L, Wang F, Wang M, Wang S, Lv J, et al. Cardiovascular disease in early stages of chronic kidney disease in a Chinese population. Journal of the American Society of Nephrology: JASN. 2006;17:2617-21.

7. Liu L, Zhao F, Yang Y, Qi LT, Zhang BW, Chen F, et al. The clinical significance of carotid intima-media thickness in cardiovascular diseases: a survey in Beijing. Journal of human hypertension. 2008;22:259-65.

8. Lang RM, Bierig M, Devereux RB, Flachskampf FA, Foster E, Pellikka PA, et al. Recommendations for chamber quantification: a report from the American Society of Echocardiography's Guidelines and Standards Committee and the Chamber Quantification Writing Group, developed in conjunction with the European Association of Echocardiography, a branch of the European Society of Cardiology. Journal of the American 
Society of Echocardiography: official publication of the American Society of Echocardiography. 2005;18:1440-63.

9. Palmon LC, Reichek N, Yeon SB, Clark NR, Brownson D, Hoffman E, et al. Intramural myocardial shortening in hypertensive left ventricular hypertrophy with normal pump function. Circulation. 1994;89:122-31.

10. Verdecchia P, Porcellati C, Zampi I, Schillaci G, Gatteschi C, Battistelli M, et al. Asymmetric left ventricular remodeling due to isolated septal thickening in patients with systemic hypertension and normal left ventricular masses. The American journal of cardiology. 1994;73:247-52.

11. Sakurai S, Ashida T, Takahashi N, Fujii J. [Effects of antihypertensive treatment on the thickening of the basal portion of the interventricular septum in essential hypertension]. Journal of cardiology. 1998;31:151-8.

12. Olafiranye O, Ibrahim M, Kamran H, Venner-Jones K, McFarlane SI, Salciccioli L, et al. Narrowed Aortoseptal Angle Is Related to Increased Central Blood Pressure and Aortic Pressure Wave Reflection. Cardiorenal medicine. 2012;2:177-83.

13. Loncaric F, Nunno L, Mimbrero M, Marciniak M, Fernandes JF, Tirapu L, et al. Basal Ventricular Septal Hypertrophy in Systemic Hypertension. The American journal of cardiology. 2020;125:1339-46.

14. Sengupta PP, Khandheria BK, Narula J. Twist and untwist mechanics of the left ventricle. Heart failure clinics. 2008;4:315-24.

15. Henein MY, O'Sullivan C, Sutton GC, Gibson DG, Coats AJ. Stress-induced left ventricular outflow tract obstruction: a potential cause of dyspnea in the elderly. Journal of the American College of Cardiology. 1997;30:1301-7.

16. Yoshitani H, Isotani A, Song JK, Shirai S, Umeda H, Jang JY, et al. Surgical as Opposed to Transcatheter Aortic Valve Replacement Improves Basal Interventricular Septal Hypertrophy. Circulation journal: official journal of the Japanese Circulation Society. 2018;82:2887-95.

17. Kiefer NJ, Salber GC, Burke GM, Chang JD, Guibone KA, Popma JJ, et al. The Impact of Basal Septal Hypertrophy on Outcomes after Transcatheter Aortic Valve Replacement. Journal of the American Society of Echocardiography: official publication of the American Society of Echocardiography. 2019;32:1416-25.

18. Al-Nasser F, Duncan A, Sharma R, O'Sullivan C, Coats AJ, Anker SD, et al. Beta-blocker therapy for dynamic leftventricular outflow tract obstruction. International journal of cardiology. 2002;86:199-205.

19. Ejiofor L, Di Nora C, Cervesato E, Cosei I, Ravasel A, Popescu BA, et al. Differences in left ventricular geometry in hypertensive African-Europeans and Caucasian patients. European journal of internal medicine. 2019;62:43-7.

20. Di Tullio MR, Zwas DR, Sacco RL, Sciacca RR, Homma S. Left ventricular mass and geometry and the risk of ischemic stroke. Stroke. 2003;34:2380-4.

21. Park CS, Park JB, Kim Y, Yoon YE, Lee SP, Kim HK, et al. Left Ventricular Geometry Determines Prognosis and Reverse J-Shaped Relation Between Blood Pressure and Mortality in Ischemic Stroke Patients. JACC Cardiovascular imaging. 2018;11:373-82.

\section{Figures}

\section{Figure 1}


Subgroup analysis of the relationship between BSH and RWT.

$\mathrm{BMI}$, body mass index; $\mathrm{BSH}$, basal septal hypertrophy; $\mathrm{CHD}$, coronary heart disease; RWT, relative wall thickness. 